\title{
EMDR Individual Protocol for Paraprofessional Use: A Randomized Controlled Trial With First Responders
}

\author{
Ignacio Jarero \\ Carolina Amaya \\ Martha Givaudan \\ Alaide Miranda \\ Latin American \& Caribbean Foundation for Psychological Trauma Research, Mexico
}

\begin{abstract}
The eye movement desensitization and reprocessing (EMDR) individual protocol for paraprofessional use in acute trauma situations (EMDR-PROPARA) is part of a project developed at the initiative of Dr. Francine Shapiro. This randomized clinical trial examined the effectiveness of the protocol administered by experienced EMDR therapists. There were 39 traumatized first responders on active duty randomly assigned to receive two 90-min sessions of either EMDR-PROPARA or of supportive counseling. Participants in the EMDR-PROPARA group showed benefits immediately after treatment, with their scores on the Short PTSD Rating Interview (SPRINT) showing further decreases at 3-month follow-up. In comparison, supportive counseling participants experienced a nonsignificant decrease after treatment and an increase in the SPRINT scores at the second follow-up. The significant difference between the two treatments provides preliminary support for EMDR-PROPARA's effectiveness in reducing severity of posttraumatic symptoms and subjective global improvement. More controlled research is recommended to evaluate further the efficacy of this intervention.
\end{abstract}

Keywords: acute trauma; EMDR; early psychological intervention; first responders; peer support

$\mathbf{T}$ he mental health and well-being of first responders is important to the individual, their family, the organization, and the wider community (Scully, 2011; Shakespeare-Finch, 2011). Mental health impairments in first responders are associated with substantial personal and public costs and can adversely affect their service to victims and patients (Kleim \& Westphal, 2011).

In the context of this article, we use the term "first responders" to refer to a heterogeneous grouping of both paid professionals and volunteers who provide critical services in emergencies (e.g., staff care peer support in large organizations). First responders' occupations have historically included law enforcement, emergency line operators, firefighters, search and rescue personnel, ambulance personnel, and emergency room staff. These positions are characterized by a high level of work demands and routine exposure to both physical and psychological traumatic stressors (Peñalba, McGuire, \& Leite, 2008).

Research has shown that exposure to traumatic stressors increases the likelihood of posttraumatic stress disorder (PTSD), other psychiatric disorders, and burnout (Haugen, Evces, \& Weiss, 2012). The most frequent trauma-related disorders in first responders are PTSD, major depressive disorder, and drug- and alcohol-related disorders (Benedek, Fullerton, \& Ursano, 2007). First responders who have not been specifically trained for disaster work showed higher PTSD prevalence rates (Guo et al., 2004).

\section{Clarification of Terms of Acute Trauma Situations}

A. Acute stress syndrome: An acute traumatic event that occurred within a few days and is represented 
by significant levels of symptoms clusters (e.g., hyperarousal, avoidance, dissociation, and intrusion) that have not subsided for at least several days.

B. Recent event: A traumatic event that occurred within a 2-to 3-month period and the participant has had a posttrauma safety period.

C. Cumulative trauma exposure memory network: Related stressful events that continued for an extended time (often more than 3 months), and the participant has not had a posttrauma safety period. This kind of trauma situation can occur in high-risk organizations personnel (e.g., military and first responders in active duty), natural or man-made disasters survivors, illegal immigrants, geopolitical crisis, and so forth.

For Jarero and Uribe (2011, 2012), acute trauma situations are not only related to a time frame (days or months) but also to a posttrauma safety period. They have argued that the lack of a posttrauma period of safety prevents the consolidation in memory of the original critical incident because the continuum of stressful events with similar information (e.g., emotions, physical sensations) does not give the statedependent traumatic memory (van der Kolk \& van der Hart, 1991) sufficient time to consolidate into an integrated whole. Thus, the memory network remains in a permanent excitatory state, sensitizing and expanding with each subsequent stressful event in this continuum like the ripples from a rock falling in the middle of a lake with the risk of posttraumatic stress disorder (PTSD) and comorbid disorders growing with the number of exposures.

\section{Treatment of Traumatized First Responders}

In areas with poor or no psychological trauma professional care (e.g., developing countries), first responders exposed to direct or secondary trauma/ compassion fatigue (Figley, 1995) in their work environments who fail to recover over time or after crisis intervention may benefit from a peer-provided early intervention treatment. According to Creamer et al. (2012),

The rationale for provision of peer-support programs often includes the goal of meeting the legal and moral duty to care for employees, as well as addressing multiple barriers to standard care like stigma, lack of time, poor access to providers, lack of trust, and fear of job repercussions. (p. 134)

\section{Acute Interventions}

Early intervention is understood to prevent later psychological distress or long-term psychological morbidity (Scully, 2011). There is considerable controversy over which type of intervention should be offered, at what time, and to whom after exposure to traumatic events (Roberts, Kitchiner, Kenardy, \& Bisson, 2010). Experts (e.g., Bisson, Roberts, \& Macho, 2003; Brewin et al., 2008) maintain that interventions should target those who are at highest risk for experiencing persistent psychological problems following exposure to potentially traumatic events. Various authors use different vocabulary and terms in their discussion of acute stress syndromes and treatment.

Crisis intervention is the term used by Everly and Mitchell (2008) to describe the "urgent psychological or behavioral care designed to first stabilize and then reduce symptoms of distress or dysfunction so as to achieve a state of adaptive functioning, or to facilitate access to a continuum of care when necessary" (p. 8). They propose that the goals of crisis intervention are (a) stabilization of psychological functioning through meeting basic physical needs, then addressing the most basic of psychological needs; (b) mitigation of psychological dysfunction distress; (c) return of acute adaptive psychological functioning; and/or (d) facilitation of access to the next level of care.

Early psychological interventions is the term used by Roberts et al. (2010) for interventions that began within the first 3 months after a traumatic event with the aim mostly of preventing PTSD or ongoing distress in those with traumatic stress symptoms, with acute stress disorder (ASD), or who are at risk for PTSD or other disorders. Other authors (e.g., Kehle et al., 2010) spoke of early mental health treatment, which is an intervention comprising psychotherapy and psychopharmacotherapy treatment. Furthermore, the U.S. Department of Veterans Affairs and Department of Defense (VA/DoD, 2010) called all treatment interventions for posttraumatic stress, acute stress reaction (ASR), ASD, acute PTSD, and chronic PTSD as management of posttraumatic stress. (See VA/DoD [2010] treatment guidelines for current recommended interventions and limitations.) As we can see in these examples, the word intervention could refer to a wide range of activities from addressing immediate physical needs to psychopharmacotherapy.

Several studies have applied a screen-and-treat approach after various traumatic events to systematically identify those who have developed acute posttraumatic symptoms and to provide early treatment (Brewin et al., 2008). VA/DoD’s (2010) Clinical Practice Guidelines for Management of Post-Traumatic Stress mentioned that early identification of PTSD and rapid referral to treatment can shorten suffering and lessen the severity of functional impairment. Early brief interventions are recommended for patients 
with significant early symptom levels as well as for those who are incapacitated by acute psychological or physical symptoms.

Different approaches are used today to treat first responder's trauma (e.g., psychoeducation, critical incident stress management, cognitive behavioral therapy (CBT), eye movement desensitization and reprocessing [EMDR]). Haugen et al. (2012) concluded that literature on treating PTSD in first responders was insufficient for making any evidencebased recommendations; they suggested randomized controlled trials (RCTs) on treatments such as EMDR. The promotion of positive mental health and psychological well-being in the workplace is recognized as a global research priority (e.g., World Health Organization [WHO], 2002).

\section{EMDR, First Responders, and EMDR Early Intervention}

EMDR is an integrative eight-phase treatment approach guided by the adaptive information processing (AIP) model (F. Shapiro, 2001). In this integrative psychotherapy, dysfunctional stored memories are considered to be the primary basis of clinical pathology not caused by organic deficit (F. Shapiro, 1989, 2001, 2007). The processing of these memories is posited to lead to resolution through the reconsolidation and assimilation within the larger adaptive memory networks (Oren \& Solomon, 2012). EMDR has established efficacy in the treatment of PTSD (American Psychiatric Association, 2004; Bisson \& Andrew, 2007) and is also applicable to a wide range of other experientially based clinical complaints (F. Shapiro, 2001; Solomon \& Shapiro, 2008). The VA/DoD (2010) designated EMDR as an "A" level treatment, described by the letter as "a strong recommendation that clinicians provide the intervention to eligible patients" (p. 6).

Literature review on treating chronic PTSD in first responders showed published EMDR case studies with police officers (Keenan \& Royle, 2007; Spates \& Burnette, 1995) and firefighters (Kitchiner, 2004); and an RCT of EMDR versus a traditional stress management program with 62 police officers (Wilson, Tinker, Becker, \& Logan, 2001).

The clinical experience and work in the field with EMDR early intervention (EEI) has been extensive (Maxfield, 2008). Results of published studies indicate that EEI is a brief intervention with rapid treatment effects that can be used in the field or emergency situations.

There is a body of research supporting the use of modified EMDR therapy protocols to treat acute trauma in both group and individual formats (Jarero, Artigas, \& Luber, 2011; E. Shapiro, 2012). The primary reason for the modifications is that memory consolidation appears to change in the weeks and months following a critical incident (F. Shapiro, 2001). See E. Shapiro (2012) for developments in the field of early psychological intervention after trauma in general and the place of early EMDR intervention in particular.

Jarero et al. (2011) have argued that EMDR early therapy intervention has a natural place in the crisis intervention and disaster mental health continuum of care context and may be a key to early intervention as a brief treatment modality. This article's authors advocated for providing acute interventions according to the unfolding phases of recovery and assessment of needs the survivors are experiencing (Solomon, 2008).

\section{The EMDR Individual Protocol for Paraprofessional Use in Acute Trauma Situations}

\section{Background}

The EMDR individual protocol for paraprofessional use in acute trauma situations (EMDR-PROPARA) is part of a project developed at the initiative of Dr. Francine Shapiro (personal communication, June 28, 2012).

We have recognized that developing countries often lack the professional resources to adequately respond to large traumatic events, and that there may be poor or no psychological trauma professional care. However, such nations often have a cadre of trained paraprofessionals who are responsible to provide interventions and treatment. There are antecedents of CBT effectively delivered by paraprofessionals (nonmental health practitioners) with outcomes comparable to professionals (e.g., Hepner et al., 2012; Montgomery, Kunik, Wilson, Stanley, \& Weiss, 2010).

Consequently, a special EMDR training program was developed to teach appropriately selected, trained, and supervised paraprofessionals on how to provide group and individual EMDR in acute trauma situations. The training began with a message emphasizing client safety by Dr. Shapiro, transmitted by the workshop trainers (EMDR Institute senior trainers of trainers). Dr. Shapiro encouraged the paraprofessionals to pay careful attention to treatment fidelity and research rigor so that data could be gathered in a systematic way and be scientifically evaluated to yield meaningful information.

For the group format, the EMDR Integrative Group Treatment Protocol (Artigas, Jarero, Alcalá, \& Lopez Cano, 2009) was selected. This protocol has been used in its original format, or with adaptations, to meet the 
circumstances in numerous settings around the world (Gelbach \& Davis, 2007; Maxfield, 2008). An overview of this protocol is in Jarero and Artigas's (2012) work.

The EMDR-PROPARA was developed for the first author (IJ) for the individual format. To ensure the protocol ecological validity (acceptability in the real world; Brewer, 2000), a field evaluation was conducted for the Latin American \& Caribbean Foundation for Psychological Trauma Research task force members in United States, Mexico, Central America, South America, and Spain. The treatment was provided to different populations in acute trauma phase: military personnel and emergency responders on duty, civilians (kidnapping), and illegal immigrants.

The EMDR-PROPARA is an adaptation for paraprofessional use of the EMDR Protocol for Recent Critical Incidents (EMDR-PRECI). This last protocol is a modification of F. Shapiro's (2001) Recent Traumatic Events Protocol provided in an individual treatment format to clients suffering from recent ongoing trauma. It was developed in the field to treat critical incidents where related stressful events continue for an extended time and where there is no posttrauma period of safety for memory consolidation (see Jarero et al., 2011 for a detailed description of the protocol).

There is preliminary evidence supporting the efficacy of EMDR-PRECI in reducing symptoms of posttraumatic stress in adults and maintaining those effects despite ongoing threat and danger in a disaster mental health continuum of postincident care context. EMDR-PRECI was evaluated in a study by Jarero et al. (2011) who compared immediate treatment and waitlist/delayed treatment groups with 18 adults who had been traumatized by a recent 7.2 magnitude earthquake in North Baja California, Mexico. Results showed that one session (lasting between 80 and $130 \mathrm{~min}$ ) of EMDR-PRECI produced significant improvement of symptoms of posttraumatic stress for both the immediate treatment and waitlist/ delayed treatment groups, with results maintained at 12-week follow-up.

An EMDR-PRECI field study investigated early intervention with traumatized Mexican first responders (law enforcement/forensic personnel) working in a human massacre situation under extreme stressors where there was no posttrauma period of safety for memory consolidation (Jarero \& Uribe, 2011, 2012). Results showed the reduction of scores on self-report measures of posttraumatic stress and PTSD symptoms, lending support to the view that EMDR can be used effectively as an early intervention for traumatized first responders. It appeared that EMDR-PRECI helped to prevent the development of chronic PTSD and to increase psychological and emotional resilience.

Development of EMDR-PROPARA. Norris, Murphy, Baker, and Perilla (2004) call for "early and ongoing interventions that provide mental health care to disaster victims in a way that is culturally appropriate and feasible for places that have few mental health professionals to draw upon" (pp. 290-291). The EMDR-PROPARA was designed for paraprofessional use. It is aimed at reducing the severity of symptoms of posttraumatic stress as well as symptoms of somatic distress, stress coping, and work, family, and social impairment. It was specifically designed to address the three clinical presentations that are common among those who fail to recover in acute trauma situations: (a) subthreshold variants of ASD or acute PTSD, (b) functional and/or social impairment, and (c) subclinical traumatic stress symptoms that impact life quality. These presentations can develop over time or after early interventions (i.e., after addressing immediate physical needs, social and spiritual support, psychoeducation, normalization, and psychological first aid). Based on the authors' field and clinical experience, these clinical presentations could be derived from the three acute trauma situations mentioned at the beginning of this article: acute stress syndrome, recent event, and cumulative trauma exposure memory network.

Because the application of EMDR by paraprofessionals is a new venture, the authors consider that follow-up after the EMDR-PROPARA intervention to determine patient status should be mandatory. We recommend the use of a validated, self-administered checklist (e.g., SPRINT) to ensure systematic, standardized, and efficient review of the patient's symptoms and history of trauma exposure. Routine ongoing use of these checklists may allow assessment of treatment response and patient progress (VA/DoD, 2010). The quality of care provided by the paraprofessional and the paraprofessional itself must be carefully monitored through supervision in a noncritical positive context.

Patients who do not improve or whose status worsens should be referred to evidence-based psychotherapy (e.g., EMDR or trauma-focused CBT). In areas with poor or no psychological trauma professional care, consider the use of adjunctive treatment with alternative care modalities such as complementary alternative medicine with mind-body treatments (e.g., acupuncture, meditation, yoga, relaxation), consistent with available resources and resonant with patient belief systems (Strauss \& Lang, 2012). 


\section{Method}

The Latin American \& Caribbean Foundation for Psychological Trauma Research review board approved the research protocol. Participants gave written informed consent prior to enrollment. The purpose of research was to assess the effectiveness of EMDRPROPARA as an intervention for a recent traumatic event. Consequently, during assessment and treatment, all participants were asked to focus on the worst work experience in the past 3 months. To measure the impact of the EMDR-PROPARA, a pre-post design was carried out to compare EMDR-PROPARA and supportive counseling. Four measurement occasions enabled exploration of longer treatment effects.

\section{Participants}

Thirty-nine first responders in active duty (20 male, 19 female) from Sonora, Mexico were voluntarily recruited from Red Cross paramedics $(n=15)$, emergency line operators $(n=15)$, and firefighters $(n=9)$. The participants were randomized to receive supportive counseling $(n=20)$ or EMDR-PROPARA $(n=19)$. Inclusion criteria included the following: (a) to be first responders, (b) to be on active duty, and (c) aged 18-60 years. Exclusion criteria included (a) current suicidal ideation; (b) a diagnosis of psychotic or bipolar disorder, organic mental disorder, or substance abuse; (c) current suicidal or homicidal ideation; and (d) significant cognitive impairment. All participants continued in active duty during the study duration. Their attendance in treatment was not mandated by the employer and there were no dropouts in this study.

\section{Measures}

The SPRINT is an eight-item interview or self-rating questionnaire with solid psychometric properties that can serve as a reliable, valid, and homogeneous measurement of PTSD illness severity and global improvement as well as a measure of somatic distress, stress coping, and work, family, and social impairment. Each item is rated on a 5-point scale: not at all (0), a little bit (1), moderately (2), quite a lot (3), and very much (4). Scores between 18 and 32 correspond to marked or severe PTSD symptoms, 11 and 17 to moderate symptoms, 7 and 10 to mild symptoms, scores of 6 or less indicated either no or minimal symptoms. The SPRINT also contains two additional items to measure global improvement according to percentage change and by severity rating. This questionnaire was translated from English to Spanish, then translated back from Spanish to English, and reviewed and authorized by one of its authors. SPRINT performs similarly to the Clinician-Administered PTSD Scale (CAPS) rating scale in the assessment of PTSD symptoms clusters and total scores and can be used as a diagnostic instrument (Vaishnavi, Payne, Connor, \& Davidson, 2006). In the SPRINT, a cutoff score of 14 or more was found to carry a $95 \%$ sensitivity to detect PTSD and $96 \%$ specificity for ruling out the diagnosis, with an overall accuracy of correct assignment being 96\% (Connor \& Davidson, 2001).

\section{Procedure}

Recruitment occurred from August 6 to 30, 2012. Recruitment involved a two-stage process: (a) Researchers met with potential participants at their institutions and explain the study with the inclusion and exclusion criteria; and (b) written informed consent was obtained from the volunteers. Demographic information was also collected.

Participants were assessed with the SPRINT before treatment (Time 1), after treatment (Time 2), at 1-month follow-up (Time 3), and at 3-month follow-up (Time 4). All posttreatment and follow-up assessments were conducted by a professional who was blind to treatment group status.

\section{Treatments}

Participants were randomized to supportive counseling group $(n=20)$ or EMDR-PROPARA group $(n=19)$. Each group received two 1.5 -hr individually administered sessions conducted by one of the four EMDR clinicians working in this study (one EMDR Institute trainer of trainers, one EMDR-approved consultant, and two EMDR-certified therapists). Each EMDR therapist was trained in the EMDR-PROPARA by the first author. Treatment adherence was facilitated by strict compliance with therapy protocols.

EMDR-PROPARA. The EMDR-PROPARA has the following phases:

1. Working place preparation

2. History: (a) gathering of demographic information; (b) asking about the critical incident; (c) physical, mental, emotional, and behavioral symptoms assessment; and (d) instrument administration

3. Participant preparation: self-soothing techniques

4. Facilitation of traumatic memory processing: (a) assessment of traumatic memory, (b) desensitization, (c) installation/positive self-belief enhancement, and (d) body scan

5. Session closure

6. Stress management information 
7. Reevaluation: (a) previous treatment session evaluation and (b) restarting a session

8. Reevaluation before termination: (a) present stimuli, (b) future template, (c) symptom resolution, (d) posttraumatic growth, and (e) instrument administration

Main difference between the EMDR-PRECI and the EMDR-PROPARA is the bilateral stimulation (BLS) during the traumatic memory processing. In the EMDR-PRECI, eye movement is the first BLS option. In the EMDR-PROPARA, the butterfly hug $(\mathrm{BH})$ is the only method used. Artigas and Jarero (in press) thought that the control obtained by clients over their stimulation using the $\mathrm{BH}$ may be an empowering factor that aids their retention of a sense of safety while processing traumatic memories. The authors' assumption is that during the $\mathrm{BH} / \mathrm{BLS}$, the AIP system (Shapiro, 2001) is regulating the stimulation to maintain clients in their window of tolerance (Corrigan, Fisher, \& Nutt, 2011) and allow appropriate reprocessing. According to Shapiro, the intrinsic information processing system and the client's own associative memory networks are the most effective and efficient means to achieve optimal clinical effects.

Supportive Counselling. Supportive counselling sessions included education about trauma, general problem-solving skills, and the provision of an unconditionally supportive role for the therapist. Supportive counselling specifically avoided exposure or anxiety management techniques. This psychological intervention was selected for comparison because it has been repeatedly used in RCTs to evaluate the efficacy of trauma-focused CBT as an early psychological intervention in the treatment of acute traumatic stress symptoms (e.g., Bryant, Harvey, Sackville, Dang, \& Basten, 1998; Bryant, Sackville, Dang, Moulds, \& Guthrie, 1999; Foa, Rothbaum, Riggs, \& Murdock, 1991; Roberts et al., 2010).

\section{Statistical Analysis}

Given the design of the study, we specified a repeated measures general linear model (GLM) for evaluating the effects of the EMDR-PROPARA on the SPRINT scores. The two major reasons to use repeated measures were (a) to increase statistical power and (b) to know the effect of the treatment over time. Besides the GLM, $t$ tests were conducted to compare the scores at each evaluation time.

\section{Results}

Data shows clear effects of EMDR-PROPARA in reducing symptoms of posttraumatic stress and maintaining a continuous decrease despite ongoing exposure to similar stressful events related with their daily work.

Participants were assessed with the SPRINT on four occasions: baseline (Time 1), posttreatment (Time 2), at 1-month follow-up (Time 3), and at 3-month follow-up (Time 4). Figure 1 shows the significant overall difference between supportive counseling

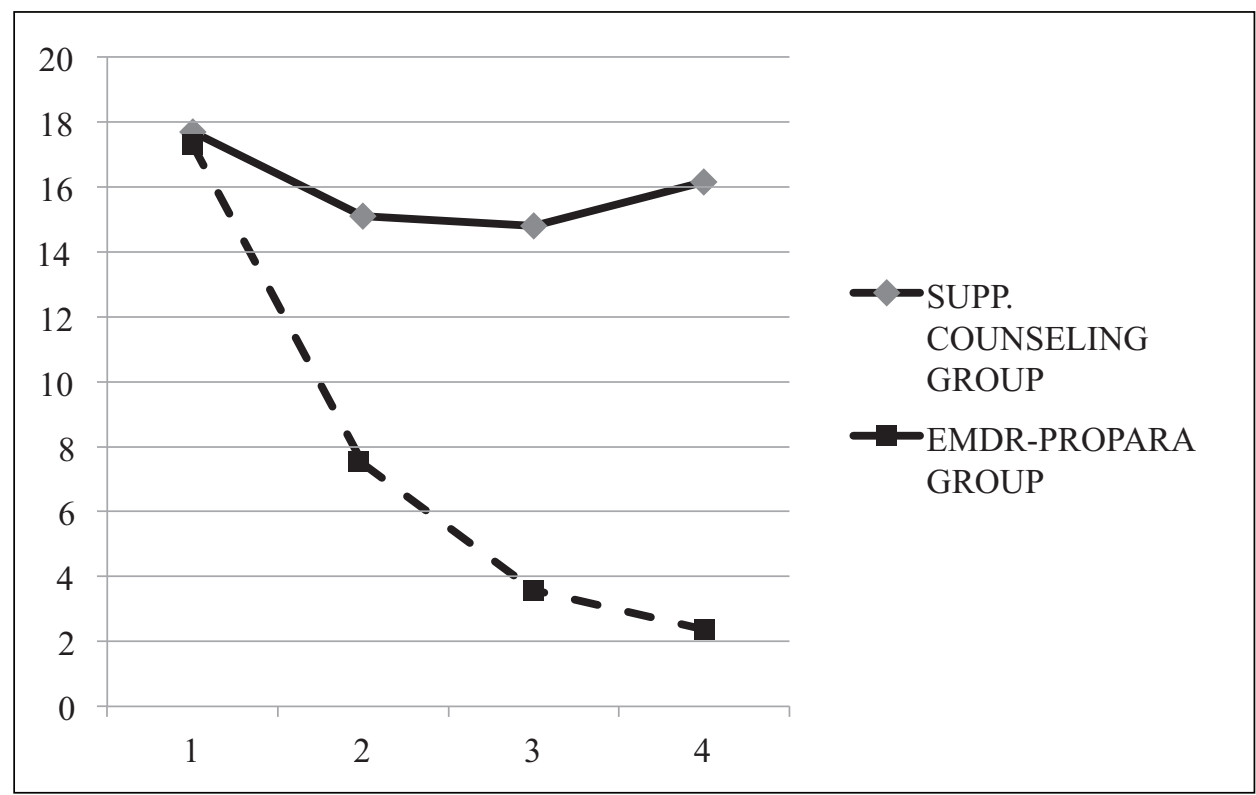

FIGURE 1. Mean Short PTSD Rating Interview (SPRINT) scores. Time $1=$ baseline, Time 2 $=$ posttreatment, Time $3=1$-month follow-up, and Time $4=3$-month follow-up. Supp $=$ supportive; EMDR-PROPARA = eye movement desensitization and reprocessing individual protocol for paraprofessional use in acute trauma situations. 
TABLE 1. Means (SD) and Statistical Comparisons for SPRINT Scores

\begin{tabular}{|c|c|c|c|c|}
\hline \multirow[b]{2}{*}{ Time } & \multicolumn{2}{|c|}{ Mean $(S D)$} & \multicolumn{2}{|c|}{$\begin{array}{c}\text { Statistical } \\
\text { Comparison }\end{array}$} \\
\hline & $\begin{array}{l}\text { Supportive } \\
\text { Counseling } \\
\quad(n=20)\end{array}$ & $\begin{array}{l}\text { EMDR- } \\
\text { PROPARA } \\
(n=19)\end{array}$ & $t(37)$ & $p$ \\
\hline Baseline & $17.70(4.94)$ & $17.26(4.41)$ & 0.299 & .767 \\
\hline Posttreatment & $15.10(4.59)$ & $7.47(2.54)$ & 6.35 & .001 \\
\hline $\begin{array}{l}\text { First } \\
\text { follow-up }\end{array}$ & $14.80(5.34)$ & $3.58(1.61)$ & 8.77 & .001 \\
\hline $\begin{array}{l}\text { Second } \\
\text { follow-up }\end{array}$ & $16.15(3.92)$ & $2.36(0.83)$ & 14.98 & .001 \\
\hline
\end{tabular}

Note. SPRINT = Short PTSD Rating Interview; EMDRPROPARA $=$ EMDR individual protocol for paraprofessional use in acute trauma situations.

and EMDR-PROPARA. Table 1 shows mean scores and standard deviations for both groups in the four periods.

\section{Group Equivalence at Baseline}

The mean SPRINT scores for participants (EMDRPROPARA: $\chi^{2}=17.26, S D=4.13$; supportive counseling: $\chi^{2}=17.70, S D=4.93$ ) were in the moderate range and higher than the PTSD cutoff of 14 (see Table 1). There was no significant difference between the baseline scores of the two treatment groups, $t(37)$ $=0.299, p=.76$.

\section{Treatment Effects Across Time}

Results of the repeated measures GLM found a significant overall difference between supportive counseling and EMDR-PROPARA groups over time, $F(1,35)=$ 138.97, $p=.001$. Results confirm the main effects of group and the main effects of time over the four measures. Between-subjects effects showed differences between supportive counseling and EMDR-PROPARA group, $F(1,35)=92.29, p=.001$. Interaction between sex and group showed no significant differences, $F(1,35)=1.260, p=269$, indicating that men and women have the same pattern of response. Within-subjects comparison demonstrated a significant interaction of time and group, $F(1,35)=524.87$, $p=.001$. This effect can be seen in Figure 1, which illustrates the consistent decrease in the mean scores of the EMDR-PROPARA group.

\section{Short-Term Treatment Effect}

An independent sample $t$ test was used to compare the posttreatment SPRINT scores for EMDR-PROPARA and supportive counseling. The SPRINT score for the EMDR-PROPARA group was significantly smaller than that of supportive counseling, $t(37)=6.35, p=$ .001 (see Table 1). Results show the immediate effectiveness of EMDR-PROPARA.

\section{Maintenance of Treatment Effect}

A statistical analysis using $t$ test for independent samples compared the SPRINT scores for EMDR-PROPARA and supportive counseling at each of the two follow-up assessments (see Table 1). On each occasion, the mean of the EMDR-PROPARA group was significantly lower than those of the supportive counseling group as was expected.

\section{Subjective Global Improvement}

The SPRINT contains two items to measure global improvement. Item 1 assesses percentage change asking, "How much better do you feel since beginning treatment? As a percentage between 0 and 100." Item 2 rates symptom severity, asking the respondent to use a 5 -point Likert scale (where $1=$ worse, $2=$ no change, $3=$ minimally, $4=$ much, and $5=$ very much) asking, "How much have the above symptoms improved since starting treatment?" On Item 1 , the mean response at follow-up for the EMDRPROPARA group was $91 \%$; and on Item 2 , the mean response at follow-up was very much. These results showed subjective global improvement for this group. Conversely, on Item 1, the mean response at follow-up for the supportive counseling group was $63 \%$; and on Item 2 , the mean response at follow-up was minimally.

\section{Discussion}

This study examined the effectiveness of the EMDRPROPARA - an individual treatment protocol designed for paraprofessional use in acute trauma. Participants in the EMDR-PROPARA group showed benefits immediately after treatment, with their scores on the SPRINT showing further decreases at the second follow-up conducted 3 months posttreatment. In comparison, supportive counseling controls experienced a nonsignificant decrease after treatment and an increase in the SPRINT scores at the second follow-up. Results provide preliminary support for EMDR-PROPARA's effectiveness in reducing severity of posttraumatic symptoms and subjective global improvement. 
At baseline, the average score for both treatment groups was higher than 14-a score that is considered the cutoff for PTSD. Scores for the supportive counseling group across the four measures never decreased lower than 14, whereas in the EMDR-PROPARA group, the mean score in the posttreatment assessment was 7.47 .

Data collected during follow-up measurements confirmed that participants in both groups continued with their work activities in the same way they were used to do it before treatment. No relevant changes in participants' living conditions were reported during the study. Consequently, the study results cannot be attributed to changes in the work environment or other different stressful events. Furthermore, no preexisting psychological problems or other risk factors rendering them more vulnerable to develop PTSD were found in either of the groups that could explain differences between the two groups.

\section{Long-Term Effects of Trauma and Benefits of Treatment}

The differences between the EMDR-PROPARA and the supportive counseling group were maintained throughout the study immediately after the treatment sessions. This pattern is consistent with research investigating the longitudinal effect of EMDR treatment in subjects that are permanently exposed to stressful events because of their work circumstances (Jarero \& Uribe, 2012) and are therefore more likely to develop chronic PTSD (Orcutt, Erickson, \& Wolfe, 2004).

The EMDR-PROPARA intervention could be a key factor in protecting first responders from psychopathology, providing a rapid brief treatment effectively reducing acute symptoms so that more intensive or hard-to-access treatments such as prolonged exposure or medication would be unnecessary. In situations where the number of victims suffering from trauma is high and the number of experienced trauma clinicians is limited, EMDR-PROPARA could also function as a triage system allowing the direction of more intense therapeutic efforts only to the resistant patients that are more likely to be at risk for developing chronic syndromes.

Given that the community relies on the first responder's service in times of crisis, research on trauma among this special population becomes of paramount importance. We agreed with Roberts et al. (2010) who recommend more research to identify the most effective ways of providing psychological help in the early stages after a traumatic event.

\section{References}

American Psychiatric Association. (2004). Practice guidelines for the treatment of patients with acute stress disorder and posttraumatic stress disorder. Arlington, VA: Author.

Artigas, L., \& Jarero, I. (in press). The butterfly hug. In M. Luber (Ed.), Implementing EMDR early mental health interventions for man-made and natural disasters: Models, scripted protocols and summary sheets. New York, NY: Springer.

Artigas, L., Jarero, I., Alcalá, N., \& Lopez Cano, T. (2009). The EMDR Integrative Group Treatment Protocol (IGTP). In M. Luber (Ed.), Eye movement desensitization and reprocessing (EMDR) scripted protocols: Basic and special situations (pp. 279-288). New York, NY: Springer.

Benedek, D. M., Fullerton, C., \& Ursano, R. J. (2007). First responders: Mental health consequences of natural and human-made disasters for public health and public safety workers. Annual Review of Psychology, 28, 55-68.

Bisson, J., \& Andrew, M. (2007). Psychological treatment of post-traumatic stress disorder (PTSD). Cochrane Database of Systematic Reviews, 18(3), CD003388. http:// dx.doi.org/10.1002/14651858.CD003388.pub3

Bisson, J. I., Roberts, N., \& Macho, G. S. (2003). Service innovations: The Cardiff traumatic stress initiative: An evidence based approach to early psychological intervention following traumatic events. Psychiatric Bulletin, 27(4), 145-147.

Brewer, M. B. (2000). Research design and issues of validity. In H. T. Reis \& C. M. Juss (Eds.), Handbook of research methods in social and personality psychology (pp. 3-15). Cambridge, United Kingdom: Cambridge University Press.

Brewin, C. R., Scragg, P., Robertson, M., Thompson, M., d'Ardenne, P., \& Ehlers, A. (2008). Promoting mental health following the London bombings: A screen and treat approach. Journal of Traumatic Stress, 21, 3-8.

Bryant, R. A., Harvey, A. G., Sackville, T., Dang, S. T., \& Basten, C. (1998). Treatment of acute stress disorder: A comparison of cognitive behavioural therapy and supportive counseling. Journal Consult Clinical Psychology, 66, 862-866.

Bryant, R. A., Sackville, T., Dang, S. T., Moulds, M., \& Guthrie, R. (1999). Treating acute stress disorder: An evaluation of cognitive behavioural therapy and supportive counseling techniques. American Journal of Psychiatry, 156, 1780-1786.

Connor, K. M., \& Davidson, J. R. T. (2001). SPRINT: A brief global assessment of post-traumatic stress disorder. International Clinical Psychopharmacology, 16(5), 279-284.

Corrigan, F. M., Fisher, J. J., \& Nutt, D. J. (2011). Autonomic dysregulation and the Window of Tolerance model of the effects of complex emotional trauma. Journal of Psychopharmacology, 25(1), 17-25.

Creamer, M. C., Varker, T., Bisson, J., Darte, K., Greenberg, N., Lau, W., . . . Forbes, D. (2012). Guidelines for peer support in high-risk organizations: An international consensus study using the Delphi method. Journal of Traumatic Stress, 25, 134-141. 
Everly, G. S., Jr., \& Mitchell, J. T. (2008). Integrative crisis intervention and disaster mental health. Ellicott City, MD: Chevron.

Figley, C. E. (1995). Compassion fatigue: Coping with secondary stress in those who treat the traumatized. New York, NY: Brunner/Mazel.

Foa, E. B., Rothbaum, B. O., Riggs, D. S., \& Murdock, T. B. (1991). Treatment of posttraumatic stress disorder in rape victims: A comparison between cognitive-behavioural procedures and counseling. Journal Consult Clinical Psychology, 59, 715-723.

Gelbach, R., \& Davis, K. (2007). Disaster response: EMDR and family systems therapy under communitywide stress. In F. Shapiro, F.W. Kaslow, \& L. Maxfield (Eds.), Handbook of EMDR and family therapy processes (pp. 387-406). New York, NY: John Wiley \& Sons.

Guo, U., Chen, C., Lu, M., Tan, H. K., Lee, H., \& Wang, T. (2004). Posttraumatic stress disorder among professional and nonprofessional rescuers involved in an earthquake in Taiwan. Psychiatry Research, 127, 35-41.

Haugen, P. T., Evces, M., \& Weiss, D. S. (2012). Treating posttraumatic stress disorder in first responders: A systematic review. Clinical Psychology Review, 32, 370-380.

Hepner, K. A., Miranda, J., Woo, S., Watkins, K. E., Lagomasino, I., Wiseman, S. H., \& Munoz, R. F. (2012). Building recovery by improving goals, habits, and thoughts (BRIGHT): A group cognitive behavioral therapy for depression in clients with co-occurring alcohol and drug use problems. Group member's workbook. Santa Monica, CA: RAND Corporation. Retrieved from http: / / www.rand. org/pubs/technical_reports/TR977z2

Jarero, I., \& Artigas, L. (2012). The EMDR Integrative Group Treatment Protocol: EMDR group treatment for early intervention following critical incidents. European Review of Applied Psychology, 62, 219-222.

Jarero, I., Artigas, L., \& Luber, M. (2011). The EMDR protocol for recent critical incidents: Application in a disaster mental health continuum of care context. Journal of EMDR Practice and Research, 5(3), 82-94.

Jarero, I., \& Uribe, S. (2011). The EMDR protocol for recent critical incidents: Brief report of an application in a human massacre situation. EMDR Practice and Research, 5(4), 156-165.

Jarero, I., \& Uribe, S. (2012). The EMDR protocol for recent critical incidents: Follow-up report of an application in a human massacre situation. EMDR Practice and Research, 6(2), 50-61.

Keenan, P., \& Royle, I. (2007). Vicarious trauma and first responders: A case study utilizing eye movement desensitization and reprocessing (EMDR) as the primary treatment modality. International Journal of Emergency Mental Health, 9, 291-298.

Kehle, S. M., Polusny, M. A., Murdoch, M., Erbes, C., Arbisi, P., Thuras, P., \& Meis, L. (2010). Early mental health treatment-seeking among U.S. National Guard soldiers deployed to Iraq. Journal of Traumatic Stress, 23(1), 33-40.
Kitchiner, N. J. (2004). Psychological treatment of three urban firefighters with posttraumatic stress disorder using eye movement desensitization and reprocessing (EMDR) therapy. Complementary Therapies in Nursing \& Midwifery, 10, 186-193.

Kleim, B., \& Westphal, M., (2011). Mental health in first responders: A review and recommendation for prevention and intervention strategies. Traumatology, 17(4), $17-24$.

Maxfield, L. (2008). EMDR treatment of recent events and community disasters. Journal of EMDR Practice \& Research, 2(2), 74-78.

Montgomery, E. C., Kunik, M. E., Wilson, N., Stanley, M. A., \& Weiss, B. (2010). Can paraprofessionals deliver cognitive-behavioral therapy to treat anxiety and depressive symptoms? Bulletin of the Menninger Clinic, 74(1), 45-62.

Norris, F. H., Murphy, A. D., Baker, C. K., \& Perilla, J. L. (2004). Postdisaster PTSD over four waves of a panel study of Mexico's 1999 flood. Journal of Traumatic Stress, 17(4), 283-292.

Orcutt, H. K., Erickson, D. J., \& Wolfe, J. (2004). The course of PTSD symptoms among Gulf War veterans: A growth mixture modeling approach. Journal of Traumatic Stress, 17(3), 195-202.

Oren, E., \& Solomon, R. (2012). EMDR therapy: An overview of its development and mechanisms of action. European Review of Applied Psychology, 62, 197-203.

Peñalba, V., McGuire, H., \& Leite, J. R. (2008). Psychosocial interventions for prevention of psychological disorders in law enforcement officers. Cochrane Database of Systematic Reviews, (3), CD005601.

Roberts, N. P., Kitchiner, N. J., Kenardy, J., \& Bisson, J. I. (2010). Early psychological interventions to treat acute traumatic stress symptoms. Cochrane Database of Systematic Reviews, (3), CD007944.

Scully, P. J. (2011). Taking care of staff: A comprehensive model of support for paramedic and emergency medical dispatchers. Traumatology, 17(4), 35-42.

Shakespeare-Finch, J. (2011). Primary and secondary trauma in emergency personnel. Traumatology, 17(4), $1-2$.

Shapiro, E. (2012). EMDR and early psychological intervention following trauma. European Review of Applied Psychology, 62, 241-251.

Shapiro, F. (1989). Efficacy of the eye movement desensitization procedure in the treatment of traumatic memories. Journal of Traumatic Stress Studies, 2, 199-223.

Shapiro, F. (2001). Eye movement desensitization and reprocessing: Basic principles, protocols, and procedures (2nd ed.). New York, NY: Guilford Press.

Shapiro, F. (2007). EMDR, adaptive information processing, and case conceptualization. Journal of EMDR Practice and Research, 1, 68-87.

Solomon, R. (2008). Critical incident interventions. Journal of EMDR Practice and Research, 2, 160-165. 
Solomon, R. M., \& Shapiro, F. (2008). EMDR and the adaptive information processing model: Potential mechanism of change. Journal of EMDR Practice and Research, 2(4), 315-325.

Spates, C. R., \& Burnette, M. M. (1995). Eye movement desensitization: Three unusual cases. Journal of Behavior Therapy and Treatment Psychiatry, 26, 321-329.

Strauss, J. L., \& Lang, A. J. (2012). Complementary and alternative treatments for PTSD. PTSD Research Quarterly, 23(2), 1-7.

U.S. Department of Veterans Affairs and Department of Defense. (2010). VA/DoD clinical practice guidelines for management of post-traumatic stress. Version 2.0. Retrieved from http://http://www.healthquality. va.gov/ptsd/CPG_Summary_FINAL_MgmtofPTSD final.pdf

Vaishnavi, S., Payne, V., Connor, K., \& Davidson, J. R. (2006). A comparison of the SPRINT and CAPS assessment scales for posttraumatic stress disorder. Depression and Anxiety, 23(7), 437-440.

van der Kolk, B. A., \& van der Hart, O. (1991). The intrusive past: The flexibility of memory and the engraving of trauma. American Imago, 48(4), 425-454.
Wilson, S. A., Tinker, R. H., Becker, L. A., \& Logan, C. R. (2001). Stress management with law enforcement personnel: A controlled outcome study of EMDR versus a traditional stress management program. International Journal of Stress Management, 8, 179-200.

World Health Organization. (2002). The World Health Report 2002: Reducing risks, promoting healthy life. Geneva, Switzerland: Author.

Acknowledgments. To Dr. Francine Shapiro for her invaluable guidance and support. To the task force members that tested the protocol ecological validity in the field: Carolina Amaya, María Alicia Cavazos, Martha Givaudan, Cristina Jarero, Alaide Miranda, Shaila Romero, Georgina Sánchez, Susana Uribe, and Dinorah Villalobos. To Carolina Amaya and Monica Ponzanelli for their paramedics and police officers surveys, and to Cristina Jarero for her assistance in the English review.

Correspondence regarding this article should be directed to Ignacio Jarero, PhD, EdD, Boulevard de la Luz 771, Jardines del Pedregal, Álvaro Obregón, México City 01900. E-mail: nacho@amamecrisis.com.mx 